\title{
Highlights from Super-Kamiokande
}

\author{
Kimihiro OKUMURA ${ }^{1,2, a}$ \\ ${ }^{1}$ Research Center for Cosmic Neutrinos, Institute for Cosmic Ray Research, University of Tokyo, Kashiwa, \\ Chiba 277-8582, Japan \\ ${ }^{2}$ Kavli Institute for the Physics and Mathematics of the Universe (WPI), The University of Tokyo Institutes for \\ Advanced Study, University of Tokyo, Kashiwa, Chiba 277-8582, Japan
}

\begin{abstract}
Recent results from Super-Kamiokande experiment are reviewed in this paper; Neutrino mass hierarchy and CP violation in the lepton sector are investigated via $v_{\mu} \rightarrow v_{e}$ oscillation of the atmospheric neutrinos. The event rate, correlation with solar activity, energy spectrum of the solar neutrinos are measured via electron elastic scattering interactions. Neutrino emission from the WIMP annihilation at the center of the Sun are searched in the GeV energy regions. New project, SK-Gd project, to enhance anti-neutrino identification capability, has been approved inside the collaboration group.
\end{abstract}

\section{The Super-Kamiokande detector}

Figure 1 shows the schematic view of Super-Kamiokande (SK) detector. SK is a water Cherenkov detector, which size is $41.4 \mathrm{~m}$ and $39.3 \mathrm{~m}$ in height and diameter, respectively. It is located about $1000 \mathrm{~m}$ underground in Kamioka mine of Gifu prefecture, Japan. The total volume is $50 \mathrm{kton}$, and the fiducial volume for analysis is $22.5 \mathrm{kton}$. The detector is separated into the inner (ID) and the outer (OD) detectors. In the inner detector, 11129 20" photomultipliers (PMTs) are placed in grid on the detector wall to take the image of Cherenkov lights, which are produced by charged particles. In the outer detector 1885 8" PMTs are placed to detect activities coming from outside such as cosmic ray muons.

SK has been running almost 20 years from April 1996. The first phase of the experiment, SK-I, began with the original configuration of $40 \%$ photo coverage. The detector underwent an implosion accident losing about half of PMTs in 2001. The rest of PMTs are re-arranged uniformly, and SK-II resumed with $20 \%$ photo coverage. After full recovery, SK-III started with $40 \%$ coverage from 2006. The frontend electronics were upgraded in SK-IV.

SK is a multi-purpose detector with rich physics. Neutrino oscillations have been studied with solar and atmospheric neutrinos. SK also plays a role of the far detector in T2K long baseline experiment, where neutrino oscillation has been studied with accelerator neutrinos. At the same time, we are searching for astronomical neutrinos, such as supernova burst and supernova relic neutrinos. Proton decay is predicted in GUT theory, which is the physics beyond standard model. WIMP annihilation signal from astronomical objects, such as the Sun, may be observable if the mass range is in the energy range from $\mathrm{GeV}$ to $\mathrm{TeV}$.

\footnotetext{
ae-mail: okumura@icrr.u-tokyo.ac.jp
} 
This paper reviews the recent results and status of SK. It goes with atmospheric neutrino (Sec. II), solar neutrino (Sec. III), solar WIMP search (Sec. IV), SK-Gd project (Sec. V), and summary (Sec. $\mathrm{VI})$.

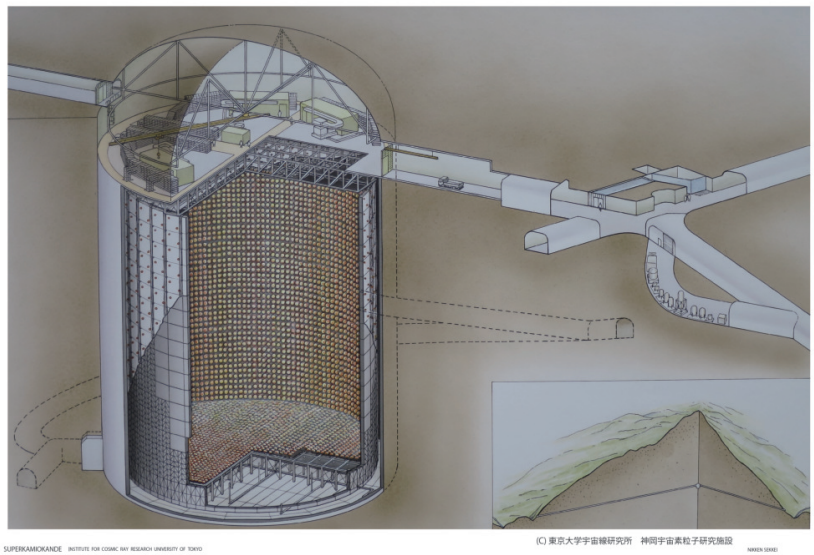

Figure 1. Schematic drawing of Super-Kamiokande detector.

Table 1. Experimental phase, observation period, and detector configuration of Super-Kamiokande

\begin{tabular}{lcc}
\hline \hline Phase & Period & \# of ID PMTs (photo coverage) \\
\hline SK-I & Apr. 1996 - Jul. 2001 & $11146(40 \%)$ \\
SK-II & Oct. 2002 - Oct. 2005 & $5182(20 \%)$ \\
SK-III & Jul. 2006 - Aug. 2008 & $11129(40 \%)$ \\
SK-IV & Sep. 2008 - Present & $11129(40 \%)$ \\
\hline \hline
\end{tabular}

\section{Oscillation analysis with atmospheric neutrino}

Since the existence of the neutrino mass became evident in 1998 [1], three-flavor mixing of the neutrino oscillation is widely accepted as the standard theory. The flavor eigenstate of three neutrinos $\left(v_{\alpha}, \alpha=\mathrm{e}, \mu, \tau\right)$ are expressed by the mass eigenstate $\left(v_{i}, i=1,2,3\right)$ and PMNS [2, 3] mixing matrix as follows:

$$
\left(\begin{array}{l}
v_{e} \\
v_{\mu} \\
v_{\tau}
\end{array}\right)=\left(\begin{array}{ccc}
1 & 0 & 0 \\
0 & \cos \theta_{23} & \sin \theta_{23} \\
0 & -\sin \theta_{23} & \cos \theta_{23}
\end{array}\right)\left(\begin{array}{ccc}
\cos \theta_{13} & 0 & \sin \theta_{13} e^{-i \delta_{C P}} \\
0 & 1 & 0 \\
-\sin \theta_{13} e^{i \delta_{C P}} & 0 & \cos \theta_{13}
\end{array}\right)\left(\begin{array}{ccc}
\cos \theta_{12} & \sin \theta_{12} & 0 \\
-\sin \theta_{12} & \cos \theta_{12} & 0 \\
0 & 1 & 0
\end{array}\right)\left(\begin{array}{c}
v_{1} \\
v_{2} \\
v_{3}
\end{array}\right)
$$

According to the neutrino oscillation measurements using the atmospheric [4, 5], reactor [6-9], accelerator [10-13], solar neutrinos [14, 15], the values of two mass splitting, $\left(\Delta m_{21}^{2}, \Delta m_{32}^{2}\right)$, and three mixing angles, $\left(\theta_{12}, \theta_{23}, \theta_{13}\right)$, have been measured. However, still important pieces are missing, such as, CP violation phase, $\delta_{C P}$ and neutrino mass hierarchy. We have a great interest in measuring $\delta_{C P}$ since it may be the source of matter/anti-matter asymmetry universe. The mass hierarchy means the ordering of second and third mass state. The normal (inverted) hierarchy is defined as that mass of the 3 rd neutrino is larger (smaller) than others, as shown in Figure 2. The octant of $\theta_{23}$, i.e. $\theta_{23}>\pi / 4$ or $<\pi / 4$, is also unknown. 


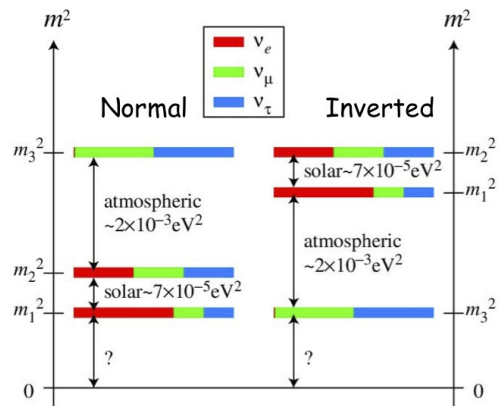

Figure 2. Scheme of normal and inverted mass hierarchy.

These parameters are possible to be probed by using atmospheric neutrinos. The atmospheric neutrinos are the decay products of secondary particles, such as pions and kaons, generated by the interactions of cosmic-ray and atmosphere. SK has been observing the atmospheric neutrino events in a wide energy range from sub-GeV up to $\mathrm{TeV}$ scale.

The oscillation of the upward-going electron neutrino, which travels in a long distance inside the Earth, is affected by the matter effect, and contains important information on the mass hierarchy and $\mathrm{CP}$ violation phase. Figure 3 shows the oscillated electron neutrino flux relative to the non-oscillated flux in the upward-going direction. A resonant-like enhancement by MSW effect is seen around multi $\mathrm{GeV}$ region. Interestingly the resonance occurs for either neutrino or anti-neutrino, depending on the mass hierarchy, i.e. the resonance occurs only for neutrino (anti-neutrino) oscillation with normal (inverted) hierarchy case. In addition $\theta_{23}$ octant affect the amplitude of the resonance. The absolute flux of the electron neutrinos in the range of Sub-GeV energy region is affected by $\delta_{C P}$ value.

A fit to SK data was performed with several oscillation parameter sets of $\delta_{C P}, \theta_{23}, \Delta m_{32}^{2}$ in the interested regions, based on $\chi^{2}$ value between data and Monte Calro (MC) expectation. This fit was also applied twice with normal and inverted hierarchy assumptions, respectively. Figure 4 shows $\chi^{2}$ difference between normal and inverted mass hierarchy assumptions. The difference of minimum $\chi^{2}$ values for each assumption was 3.0. This means that SK data favors normal hierarchy, but less than $2 \sigma$ significance. Also $\chi^{2}$ becomes minimum in $\delta_{C P} \sim 240^{\circ}$ and $\sin ^{2} \theta_{23}>0.5$.

We introduced $\mathrm{T} 2 \mathrm{~K}$ constraint into oscillation fit in order to improve mass hierarchy sensitivity since T2K data can reduce $\Delta m_{32}^{2}$ and $\theta_{23}$ uncertainties. A model of T2K predictions is constructed using public available data from T2K, and $\chi^{2}$ contribution from $\mathrm{T} 2 \mathrm{~K}$ data is calculated for each oscillation parameter. This is possible since the neutrino cross section and the detector efficiency are same due to the usage of the same detector between T2K and SK experiments, Another oscillation fit was performed including T2K constraint. As a result of the fit, normal hierarchy is still favored. Figure 5 shows the $\chi^{2}$ difference $\left(\Delta \chi^{2}\right)$ from best fit point for different data sample. Both SK and T2K favored around 280 degrees in $\delta_{C P}$, but CP conservation, which corresponds to $\delta_{C P} \sim 0$ or $\pi$, is still allowed at least $90 \%$ confidence level.

\section{Observation of solar neutrino}

SK is observing Boron 8 solar neutrino via electron scattering interaction in water. At present there are two main motivations to analyze solar neutrino. One motivation is to observe spectrum distortion due to the transition from vacuum oscillation below $1 \mathrm{MeV}$ to matter oscillation above $5 \mathrm{MeV}$. This distortion is indicated from several solar neutrino measurements, but there is no measurement observing distorted energy spectrum in a experiment. Another motivation is day-night difference of 

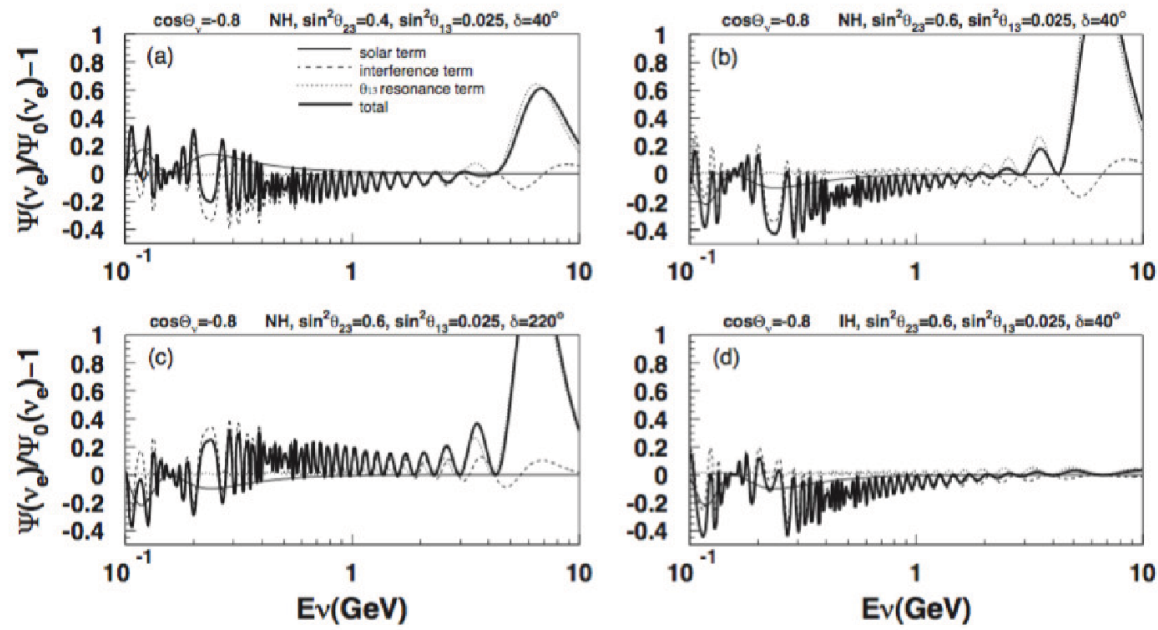

Figure 3. Oscillated $v_{e}$ flux relative to the non-oscillated flux as a function of neutrino energy for the upwardgoing neutrinos with zenith angle $\cos \theta=-0.8$. Thin solid lines, dashed lines, and dotted lines correspond to the solar term, the interference term, and the $\theta_{23}$ resonance term, respectively. Thick solid lines are total fluxes. Parameters are set as $\left(\sin ^{2} \theta_{12}, \sin ^{2} \theta_{13}, \sin ^{2} \theta_{23}, \delta_{C P}, \Delta m_{21}^{2}, \Delta m_{32}^{2}\right)=\left(0.31,0.025,0.6,40^{\circ}, 7.6 \times 10^{-5} \mathrm{eV}^{2}\right.$, $\left.+2.4 \times 10^{-} 5 \mathrm{eV}^{2}\right)$ unless otherwise noted. The $\theta_{23}$ octant effect can be seen by comparing (a) $\left(\sin ^{2} \theta_{23}=0.4\right.$ ) and (b) $\left(\sin ^{2} \theta_{23}=0.6\right) . \delta_{C P}$ value is changed to $220^{\circ}$ in (c) to be compared with $40^{\circ}$ in (b). The mass hierarchy is inverted only in (d) so $\theta_{13}$ resonance (MSW) effect disappears in this plot.
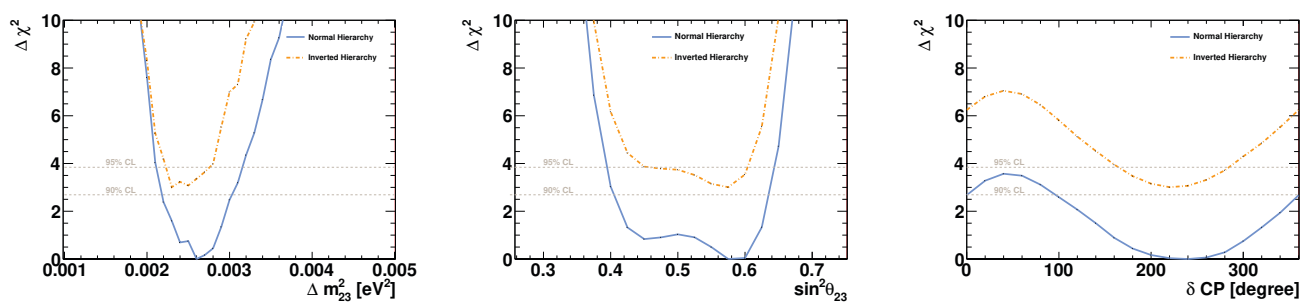

Figure 4. $\chi^{2}$ difference from the global best fit point in the oscillation fits as a function of $\Delta m_{23}^{2}$ (left), $\sin ^{2} \theta_{23}$ (middle), $\delta_{C P}$ (right), respectively. Two lines shows the cases with normal (blue solid) and inverted (orange dashed) mass hierarchy assumptions. At each point the $\chi^{2}$ is marginalized for other oscillation and nuisance parameters.

solar neutrino flux which is expected to be caused by the re-generation of Earth's matter effect when neutrino propagates the Earth.

Figure 6 shows the angular distribution of the observed events with respect to sun direction in SK-IV, and the event rate of the observed solar neutrino for each SK experiment phase. The peak due to the solar neutrino scattering is evident in the direction of the Sun. The measured event rate are consistent among the experiment phase within the uncertainties. About 77,000 events are observed from SK-I to SK-IV, and the ratio of data to unoscillated MC is calculated to be 0.4459 . 

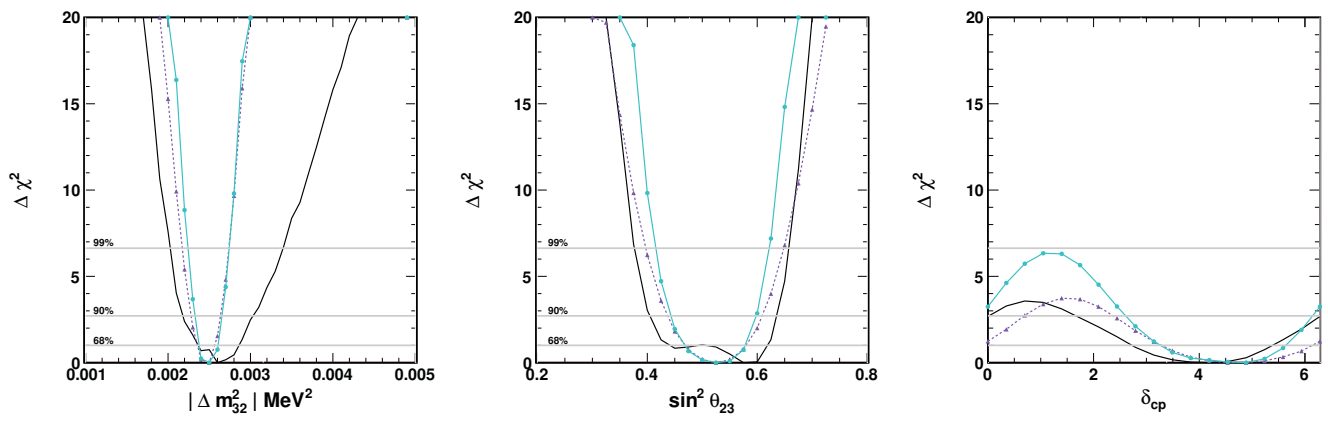

Figure 5. $\chi^{2}$ difference from the best fit point in the oscillation fits using SK (solid black), T2K model (dashed purple), and SK plus T2K model (solid light blue) data sample, as a function of $\Delta m_{23}^{2}$ (left), $\sin ^{2} \theta_{23}$ (middle), $\delta_{C P}$ (right), respectively. Normal mass hierarchy is assumed.

The yearly event rate with the Sun spot number are shown in Figure 7 from 1996 up to the present. The observed rate is consistent with flat distribution; the $p$-value of the observed rate compared with the constant is $78.6 \%$. There is no significant correlation between the solar neutrino flux and the Sun spot number,
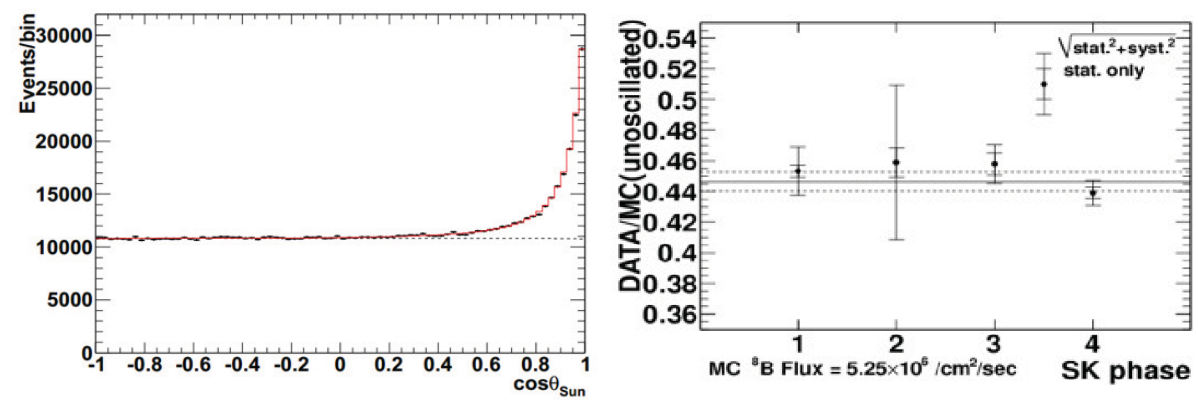

Figure 6. Angular distribution of the observed events with respect to solar direction in SK-IV (left), and the ratios of data to unoscillated Monte Carlo expectation in ${ }^{8} \mathrm{~B}$ flux for each SK experiment phase. $\cos \theta_{\text {Sun }}$ represents the cosine of the inner angle between electron direction and the solar direction. The events near the peak around $\cos \theta_{\text {Sun }}=1$ corresponds to the recoiled electron events by solar neutrino interactions, and the flat components are background.

We have been trying to reduce the background events and lower the energy threshold to observe spectrum distortion since it becomes more visible in the lower energies. The energy threshold of SK-IV is $3.5 \mathrm{MeV}$ in recoil electron kinetic energy, which is getting lower than previous SK phase. Figure 8 shows the combined energy spectrum for all SK periods. As a result of fit, the expected spectrum by MSW oscillation is slightly disfavored in the level of $<2 \sigma$ compared to other assumptions, such as exponential quadratic functions. The de-convoluted energy spectrum to neutrino energy are also shown in Figure 8. The obtained spectrum is consistent with flat and still distortion is not observable. 


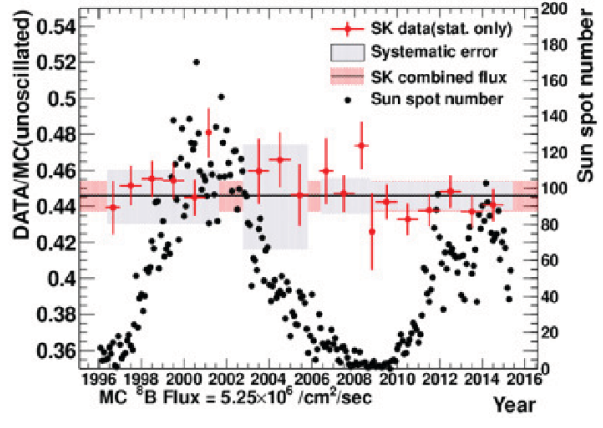

Figure 7. Ratio of data flux to unoscillated Monte Carlo expectation (red with error bar) and the Sun spot number (black dot) are shown as a function of observed date from the beginning of SK-I to the recent period of SK-IV.
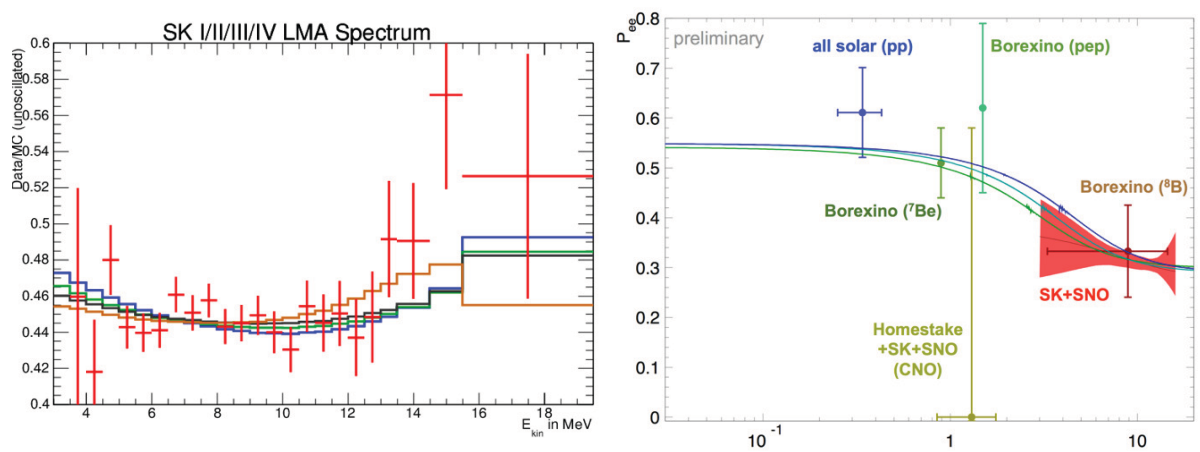

Figure 8. Combined spectrum of the recoiled electron kinematic energy from SK-I to SK-IV with the expected spectra of the various spectral assumptions (left), and the observed neutrino energy spectrum of several solar neutrino experiments (right). In the left plot the expected spectrum for the best fit oscillation parameters obtained with solar plus KamLAND (blue) and solar global (green) data are shown. The energy spectra expressed by the quadratic (black) and exponential (orange) functions are also shown. Note that the unit of the horizontal axis is the total energy of recoil electron where the detector effect such as energy resolution are not de-convoluted. The error bar represents statistical uncertainties. In the right plot the measured oscillation probability by SK plus SNO data (red) is shown with those measured with p-p solar (blue), ${ }^{7} \mathrm{Be}$ by Borexino (green), Homestake plus SK plus SNO CNO (olive), and pep by Borexino (cyan) data, as a function of neutrino energy.

\section{Search for neutrino emission from solar WIMP}

It is believed that Weakly Interacting Massive Particle (WIMPs) are attracted by the Sun's gravity, lose their energy by interactions, annihilate via $\chi \chi \rightarrow b \bar{b} / W^{+} W^{-} / \tau^{+} \tau^{-}$channels. Then neutrinos are expected to be produced as their decay products. SK is possible to search WIMP signal by looking for such neutrino events from the Sun. Recently some direct dark matter experiments, such as DAMA/LIBRA [16, 17], reported positive results in the low energy WIMP mass region around $10 \mathrm{GeV}$. We have improved our analysis to search for WIMP signal for such low energy region.

SK is suitable to search for these low mass WIMP signal. Figure 9 shows the angular distributions with respect to the Sun and the momentum distributions of several different energy samples. In the figure, the expected signal distributions are shown for the cases of $6 \mathrm{GeV} / c^{2}$ and $200 \mathrm{GeV} / c^{2}$ WIMP mass, respectively. The signal peak in the angular distributions will appear clearly in the corresponding energy range. These signals are expected to be distributed over the atmospheric neutrino backgrounds. 

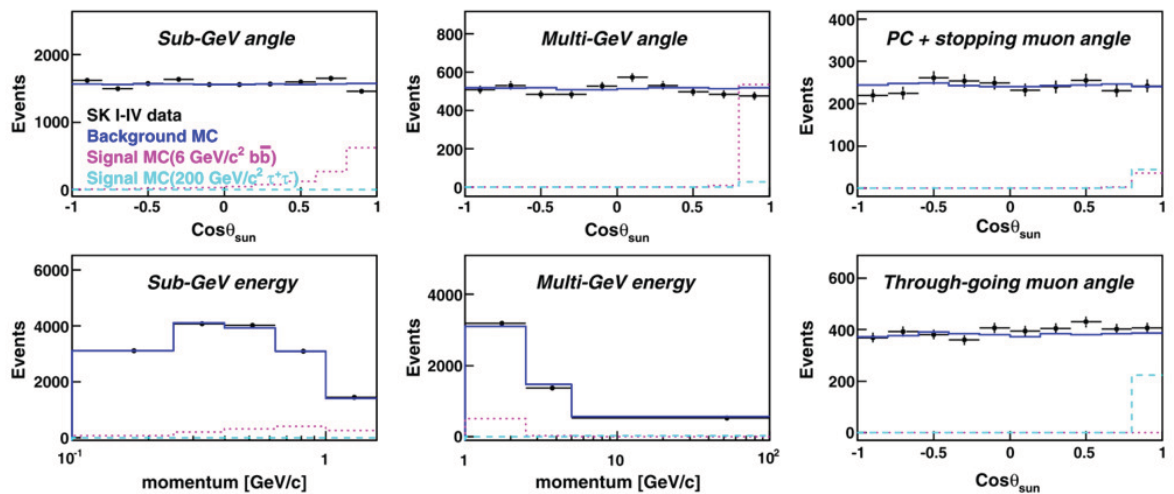

Figure 9. Angular and reconstructed momentum distributions of SK I-IV data (black crosses); atmosphericneutrino background MC (normalized to data live time, blue solid); WIMP neutrino signal MC for the $6 \mathrm{GeV} / \mathrm{c}^{2}$ $b \bar{b}$ channel (magenta dotted) $/ 200 \mathrm{GeV} / \mathrm{c}^{2} \tau^{+} \tau^{-}$channel (cyan dashed) at $90 \%$ upper limit, magnified 30 times for visibility. Among seven sub-GeV samples, the single-ring $e$-like 0 -decay-electron and $\mu$-like 0,1 -decay-electron samples are combined and shown in the left column. In the middle column, six multi-GeV samples are combined and shown. PC and up- $\mu$ samples are shown in the right column.

The WIMP signal was searched by the fitting method. The WIMP simulation are generated with several WIMP mass assumption and via the several annihilation modes of $\chi \chi \rightarrow b \bar{b}, W^{+} W^{-}, \tau^{+} \tau^{-}$. The angular distributions of the data are fitted with atmospheric MC (background) plus WIMP simulation data with varying their normalizations. As a result of fitting analysis, no significant excess was seen over the background distributions.

In order to have the meaningful upper limit on the WIMP cross section, the conversion factor from neutrino flux to WIMP cross sections is calculated using DarkSUSY 5.0.6 [18]. Also with several standard assumptions, which are commonly used in dark matter analysis, the upper limits on spin-dependent (SD) and spin-independent (SI) cross sections are obtained below $200 \mathrm{GeV} / \mathrm{c}^{2}$ in WIMP mass as shown in Figure 10 and Figure 11. The current best limits are set in SD interaction and, some fraction of WIMP allowed regions are ruled out in SI interaction. This results has been published in [19].

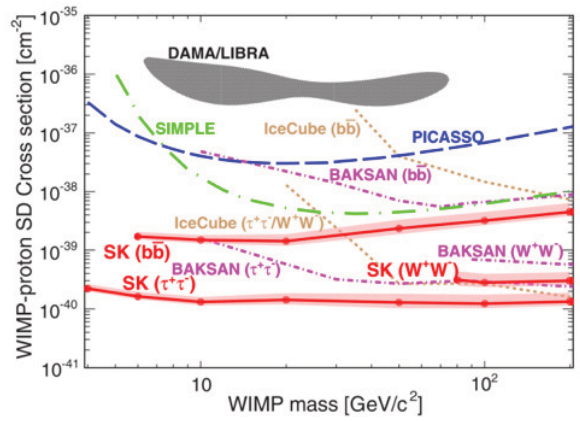

Figure 10. 90\% C. L. upper limits on SD WIMP-proton cross section calculated at DarkSUSY [18] default are shown in red solid with uncertainty bands to take account uncertainties in the capture rate for the $b \bar{b}, W^{+} W^{-}$and $\tau^{+} \tau^{-}$channels from top to the bottom. Also shown are limits from other experiments: IceCube [20] in brown dashed: (top) $b \bar{b} / W^{+} W^{-}$or $\tau^{+} \tau^{-}$ (bottom); BAKSAN in pink dot-dashed: $b \bar{b}$ (top) $/ W^{+} W^{-}$ (middle) $/ \tau^{+} \tau^{-}$(bottom); PICASSO [21] (blue long-dashed); SIMPLE [22] (green long dot-dashed). The black shaded region is the $3 \sigma$ C.L. signal claimed by

DAMA/LIBRA $[16,17]$. 


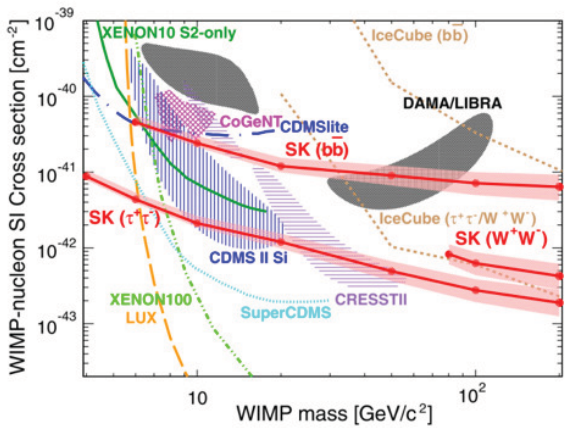

Figure 11. $90 \%$ C. L. upper limits on the SI WIMP-nucleon cross section (plotting scheme is the same as Figure 10). Also shown are event excesses or annual modulation signals reported by other experiments: DAMA/LIBRA (black shaded regions, $3 \sigma$ C.L.); CoGeNT [23] (magenta diagonally cross-hatched region, 90\% C.L.); CRESSTII [24] (violet horizontally-shaded regions, $2 \sigma$ C.L.); CDMS II Si [25] (blue vertically-shaded region, $90 \%$ C.L.); and limits: IceCube in brown dashed: $b \bar{b}$ (top) $/ W^{+} W^{-}$(middle) $/ \tau^{+} \tau^{-}$(bottom); SuperCDMS [26] (cyan dotted); CDMSlite [26] (blue long dot-dashed); XENON10 S2-only [27] (dark green dash triple dot); XENON100 [28] (green dash double dot); LUX [29] (orange long-dashed)

\section{Super-Kamiokande Gadolinium project}

SK collaboration has decided to approve new project, named as Super-Kamioande Gadolinium project (SK-Gd) in 2015 June. In the project, 0.2\% concentration of Gadolinium (Gd) sulfate is added in the pure water. When the neutrons are produced via anti-neutrino interactions, they are thermalized quickly and captured into Gd with high efficiency of about $90 \%$. The Gd atom emits multiple gammas which total energy is about $8 \mathrm{MeV}$. By detecting the prompt positron and the delayed gamma signals, the events of anti-neutrino interaction are possible to be separated from those of neutrinos.

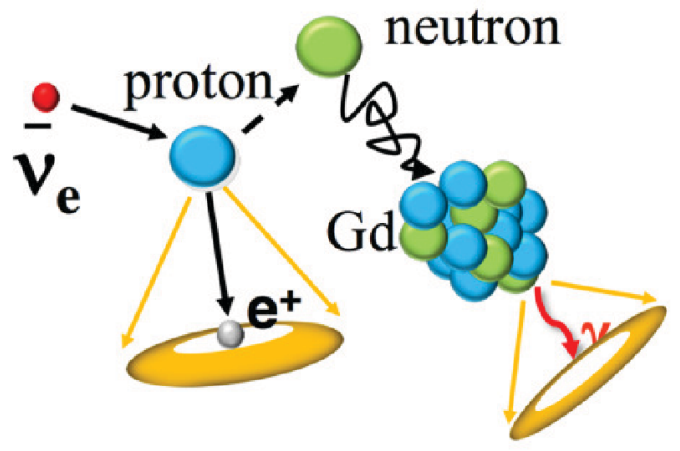

Figure 12. Schematic diagram of electron anti-neutrino reaction with Gadolinium $(\mathrm{Gd})$ water. The signal is recognized by the coincidence of the prompt positron signal and the delayed gamma produced by neutron capture with Gd.

The main motivation of this project is to detect supernova relic (SNR) neutrino, which should be produced in the past supernova. It is believed that much more star formation and supernova burst occurred in the past though such star formation rate has large uncertainties from the current astronomical observations. If the amount of SNR neutrino is measured, it will be great interest in the astrophysics and the cosmology.

The attempt in detecting SNR neutrino has also been made in current SK but not detected so far in the energy window of 10-30 MeV since the data suffers from atmospheric neutrino backgrounds, such as Michel electrons produced from low energy invisible muons. Using the coincidence technique of the prompt and the delayed signals in SK-Gd, such background events should be eliminated.

In order to investigate the feasibility of the SK-Gd project, we have been tested the effect of adding Gd in water, such as the water transparency and the material effect like rust. A small test tank of 200 $\mathrm{m}^{3}$ was build with the same detector material and photo-multipliers as used in SK to investigate the influence of $\mathrm{Gd}$ water to materials and the quality of $\mathrm{Gd}$ water. The test tank facility is shown in Figure 13. This test has been performed from October 2014 to April 2015. 
The results of the water transparency and scattering measurements by using the laser injector seems good. The amount of Cherenkov light left after $15 \mathrm{~m}$ photon travel is estimated to $92 \%$ of pure water case at $0.2 \%$ concentration of Gadolinium $(\mathrm{Gd})$ sulfate, as shown in Figure 13. Also no significant increase of the Rayleigh scattering photons, which may affect the performance of the Cherenkov light imaging, have been observed.
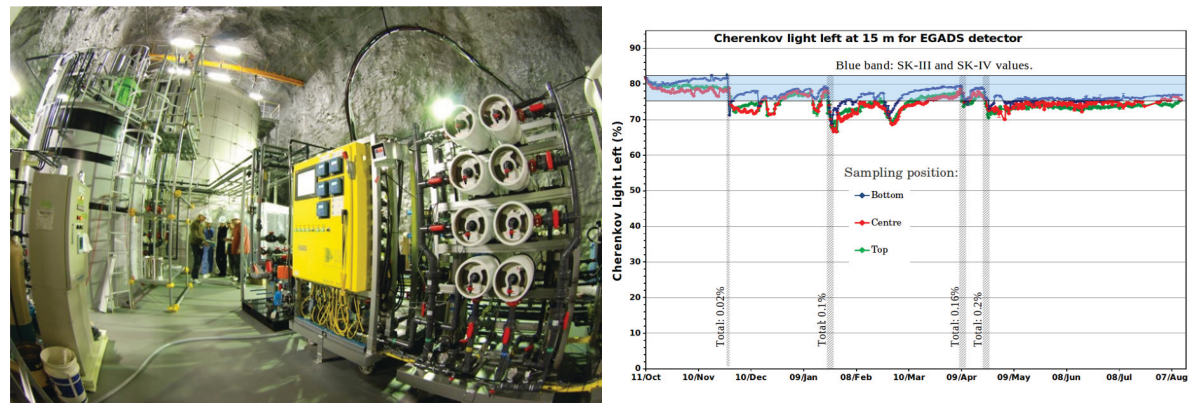

Figure 13. (Left) Photo of the EGADS tank and equipments for testing the feasibility of the Gadolinium water in SK. The $200 \mathrm{~m}^{3}$ test tank is located at the opposite side of the experimental hall. The Gd water purification system is located in the near side. (Right) The variation of the water transparency measured in EGADS tank. The measurement started with pure water, and the gadolinium sulfate is added gradually, $0.02 \%, 0.1 \%, 0.16 \%$, and $0.2 \%$. The horizontal value is shown by the expected Cherenkov light left at $15 \mathrm{~m}$ estimated based on the water transparency measured with several wavelength of the laser injector. The difference in line color represents the the different tank location (blue:bottom, red:center, green:top). The blue shaded region corresponds to those of pure water case measured in SK-III and IV.

According to these verification studies, we, SK collaboration, decided to approve SK-Gd project in 2016 June. The actual start time will be determined by considering the T2K neutrino beam schedule.

\section{Summary}

Recent physics results and the status of Super-Kamiokande experiment are reviewed in this paper. The oscillation analysis using the atmospheric neutrino shows a slight preference to normal hierarchy and $\delta_{C P} \sim 3 \pi / 2$, The expected distortion in the energy spectrum has not been seen from the solar neutrino observation. The stronger upper limits for SD and SI cross section are set in the mass range of 4-200 $\mathrm{GeV} / c^{2}$ from the indirect solar WIMP search. A new project, SK-Gd, is approved and announced by the SK collaboration, which aims to detect supernova relic neutrino using neutron capture by adding Gadolinium.

\section{References}

[1] Y. Fukuda et al. (Super-Kamiokande Collaboration), Phys. Rev. Lett. 81, 1562 (1998)

[2] Z. Maki, M. Nakagawa, S. Sakata, Prog. Theor. Phys. 28, 870 (1962)

[3] B. Pontecorvo, Sov. Phys. JETP 26, 984 (1968)

[4] Y. Ashie et al. (Super-Kamiokande), Phys. Rev. D71, 112005 (2005), hep-ex/0501064

[5] R. Wendell et al. (Super-Kamiokande Collaboration), Phys.Rev. D81, 092004 (2010), 1002.3471 
[6] S. Abe et al. (KamLAND Collaboration), Phys.Rev.Lett. 100, 221803 (2008), 0801. 4589

[7] F. An et al. (DAYA-BAY Collaboration), Phys.Rev.Lett. 108, 171803 (2012), 1203 . 1669

[8] J. Ahn et al. (RENO collaboration), Phys.Rev.Lett. 108, 191802 (2012), 1204.0626

[9] Y. Abe et al. (DOUBLE-CHOOZ Collaboration), Phys.Rev.Lett. 108, 131801 (2012), 1112.6353

[10] M. Ahn et al. (K2K), Phys.Rev. D74, 072003 (2006), hep-ex/0606032

[11] P. Adamson et al. (MINOS), Phys.Rev.Lett. 106, 181801 (2011), 1103.0340

[12] K. Abe et al. (T2K), Phys.Rev.Lett. 112, 181801 (2014), 1403.1532

[13] K. Abe et al. (T2K), Phys.Rev.Lett. 112, 061802 (2014), 1311.4750

[14] J. Hosaka et al. (Super-Kamiokande), Phys.Rev. D73, 112001 (2006), hep-ex/0508053

[15] B. Aharmim et al. (SNO), Phys.Rev. C72, 055502 (2005), nucl-ex/0502021

[16] R. Bernabei et al. (DAMA Collaboration), Eur.Phys.J. C56, 333 (2008), 0804 . 2741

[17] C. Savage, G. Gelmini, P. Gondolo, K. Freese, JCAP 0904, 010 (2009), 0808 . 3607

[18] P. Gondolo et al., JCAP 0407, 008 (2004), astro-ph/0406204

[19] K. Choi et al. (Super-Kamiokande), Phys. Rev. Lett. 114, 141301 (2015), 1503.04858

[20] M. Aartsen et al. (IceCube), Phys.Rev.Lett. 110, 131302 (2013), 1212.4097

[21] S. Archambault et al. (PICASSO Collaboration), Phys.Lett. B711, 153 (2012), 1202.1240

[22] M. Felizardo, T. Girard, T. Morlat, A. Fernandes, A. Ramos et al., Phys.Rev.Lett. 108, 201302 (2012), 1106.3014

[23] C. Aalseth et al. (CoGeNT collaboration), Phys.Rev.Lett. 106, 131301 (2011), 1002.4703

[24] G. Angloher, M. Bauer, I. Bavykina, A. Bento, C. Bucci et al., Eur.Phys.J. C72, 1971 (2012), 1109.0702

[25] R. Agnese et al. (CDMS Collaboration), Phys.Rev.Lett. 111, 251301 (2013), 1304.4279

[26] R. Agnese et al. (SuperCDMS Collaboration) (2014), 1402.7137

[27] J. Angle et al. (XENON10 Collaboration), Phys.Rev.Lett. 107, 051301 (2011), 1104.3088

[28] E. Aprile et al. (XENON100 Collaboration), Phys.Rev.Lett. 109, 181301 (2012), 1207.5988

[29] D. Akerib et al. (LUX Collaboration) (2013), 1310. 8214 